\title{
Histoire des sciences
}

\author{
Physique et minéralogie au Muséum
}

Caroline Kaspar et Philippe Jaussaud

EA 1658 LIRDHIST, Université de Lyon 1

\begin{abstract}
Plusieurs chercheurs du Muséum national d'Histoire naturelle pratiquèrent la physique entre 1793 et 1985. II en fut ainsi dans la chaire de "Minéralogie " de l'établissement, où se déroulèrent d'importants travaux : Haüy fonda la cristallographie géométrique et son élève Delafosse suivit ses traces. Des Cloizeaux appliqua l'optique à l'identification des minéraux, ainsi qu'à l'étude de leurs propriétés physiques, construisant pour cela d'ingénieux dispositifs expérimentaux. Par ailleurs Haüy, qui découvrit la piézoélectricité, exploita l'électricité et le magnétisme dans le domaine de la classification minéralogique.
\end{abstract}

La physique joua un rôle important dans I'histoire scientifique du Muséum national d'Histoire naturelle de Paris. Fondé en 1793 par un décret de la Convention, l'établissement bénéficia dès 1838 de la création d'une chaire de «Physique appliquée aux sciences naturelles », dont les premiers titulaires furent quatre membres de la famille Becquerel : AntoineCésar, Edmond, Henri - le découvreur de la radioactivité naturelle - et Jean. Ces savants éminents se succédèrent de père en fils dans les mêmes locaux vétustes du Jardin des Plantes, avant que le spécialiste de l'optique physiologique Yves Le Grand ne prenne le relais en 1949. Trente ans plus tard, le service de "Physique appliquée » du Muséum fut rebaptisé «Physico-chimie de l'adaptation biologique », pour accueillir le pharmacien militaire Pierre Douzou. Lorsque celui-ci mourut en 2000 , les chaires magistrales du Muséum n'existaient plus statutairement depuis seize ans : des départements les remplaçaient. Entre-temps, la physique avait diffusé au sein de divers laboratoires de l'établissement, comme celui d'“ Océanographie physique », ou celui de "Biophysique moléculaire " dans lequel furent étudiées les propriétés électroniques, mécaniques et optiques des macromolécules biologiques.

Une autre chaire magistrale du Muséum, celle de "Minéralogie ", participa au développement et aux applications de la physique. À ce stade de notre exposé, il est utile de rappeler qu'au $19^{\mathrm{e}}$ siècle le Muséum fut le théâtre de luttes d'influence opposant les « naturalistes » (botanistes, anatomistes ou zoologistes) aux « expérimentalistes " (physiciens, chimistes ou physiologistes) travaillant dans des chaires dites " sans collections ". Selon ces critères, le statut des minéralogistes nous apparaît aujourd'hui comme hybride : entretenant des collections, les savants concernés recouraient aussi à l'expérimentation, ainsi que nous allons l'exposer.

Son caractère ambivalent explique d'ailleurs peut-être pourquoi la chaire de "Minéralogie » du Muséum traversa les siècles, sans jamais être transformée, ni supprimée, ni rebaptisée, depuis sa création en 1793 jusqu'en 1985. Dix professeurs s'y succédèrent dont certains, comme René-Just Haüy, Gabriel Delafosse, Alfred Legrand Des Cloizeaux ou Jean Orcel, utilisèrent fréquemment les ressources de la physique pour faire progresser leurs recherches. Certains titulaires de la chaire de «Physique appliquée » subirent même l'influence de la « Minéralogie ». Ainsi, le physicien Antoine-César Becquerel découvrit avec le minéralogiste Alexandre Brongniart une vaste série de minéraux dans le sous-sol d'Auteuil. II émit aussi l'hypothèse suivante : les composés cristallisés présents dans les veines des roches s'y seraient déposés, au cours des périodes géologiques, sous l'effet de courants électriques. Enfin, la physique pure tira des bénéfices conséquents de son implication dans les travaux des minéralogistes. Cette observation est particulièrement nette, comme nous allons le voir, pour la cristallographie.

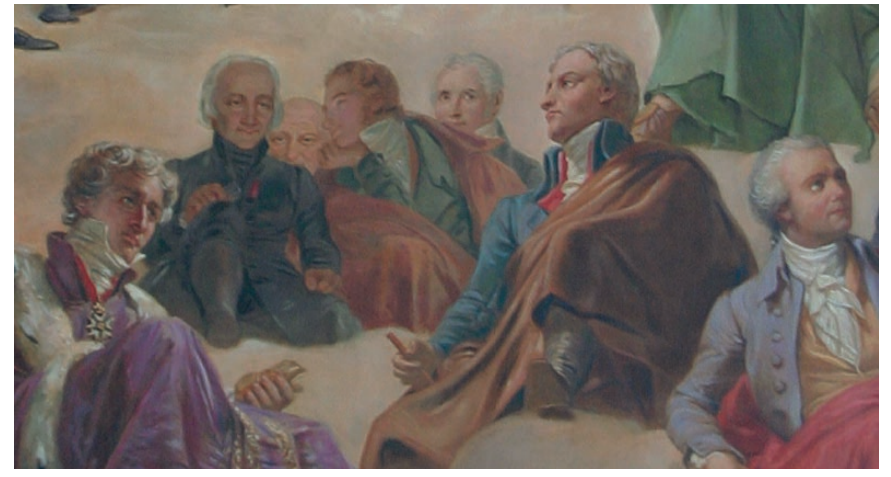

Figure 1. Détail de la fresque d'Abel Pujol intitulée "Apothéose des grands hommes qui se sont distingués dans la géologie et la minéralogie ». De gauche à droite sont représentés : Cuvier, Haüy, Dolomieu, de Saussure et Brongniart. @ École nationale supérieure des Mines de Paris.

\section{De la cristallographie géométrique à la radiocristallographie}

La naissance de la minéralogie moderne coïncide avec le développement, entre la fin du $18^{\mathrm{e}}$ et le début du $19^{\mathrm{e}}$ siècle, de la cristallographie géométrique. D'abord basée sur l'étude morphologique des cristaux naturels, celle-ci évolue rapidement vers l'analyse des lois de symétrie et l'identification des systèmes cristallins. On doit à Jean-Baptiste Romé de l'Isle la découverte de la loi de la constance des angles dièdres des cristaux : les angles entre les faces naturelles des cristaux sont constants pour une même espèce chimique. Les autres lois fondamentales de la cristallographie géométrique furent énoncées par l'abbé Haüy (1743-1822), véritable fondateur de la discipline.

Cet ancien régent du collège Cardinal Lemoine était déjà un savant reconnu lorsqu'il prit possession de la chaire de « Minéralogie » du Muséum. II avait noué à l'École des Mines une collaboration avec Nicolas Vauquelin, futur professeur de chimie au Jardin des Plantes, et les deux hommes avaient étudié ensemble divers minéraux : Haüy travaillait sur les aspects purement cristallographiques, laissant à son collègue le soin de déterminer les compositions chimiques. Des confirmations réciproques d'identités pouvaient ainsi être apportées. Selon cette logique, Haüy inspira à Vauquelin l'idée de comparer chimiquement l'émeraude et le béryl : de cette étude surgit la découverte d'un nouvel élément, le béryllium. On voit ici tout l'intérêt de l'interdisciplinarité.

En fragmentant des échantillons de spath d'Islande, Haüy - qualifié pour cela de « cristalloclaste » par Romé de l'Isle postula que les cristaux étaient des édifices constitués d'un empilement régulier de « molécules intégrantes » (voir figure 2) Le savant énonça également deux lois fondamentales de la cristallographie géométrique : celle de symétrie et celle des 
«troncatures simples » (ou des « indices rationnels »). Tous ces résultats se trouvèrent exposés dans un « Traité de minéralogie », publié en 1801, puis réédité en 1822.

Les travaux cristallographiques d'Haüy furent prolongés par l'œuvre du sixième titulaire de la chaire de " Minéralogie » du Muséum, Gabriel Delafosse (1796-1878). Reprenant la démarche de clivage des cristaux, celui-ci distingua clairement la « molécule intégrante »- qu’il rebaptisa « maille élémentaire »- de la « molécule chimique » (atome ou molécule). II établit aussi que les " mailles élémentaires ", parallélépipédiques et juxtaposées dans le cristal, constituaient un "réseau cristallin », dont les nœuds recevaient les « molécules chimiques ». En introduisant le concept de réseau, Delafosse conférait un caractère discontinu à la structure cristalline. II fut, par ailleurs, le premier à appliquer la théorie atomique à la minéralogie, puisque les " molécules chimiques » pouvaient aussi bien être des atomes.

Ayant étudié l'influence de la composition chimique et de la symétrie moléculaire sur la cristallisation des corps, Delafosse put en déduire une classification des systèmes cristallins basée sur la structure. II fut de ceux qui adhérèrent à la notion d'isomorphisme, introduite par Eilhard Mitscherlich pour expliquer les analogies de certaines formes cristallines de compositions chimiques différentes. Notons qu'Haüy s'opposa vivement à Mitscherlich sur ce point. Enfin, Delafosse établit une classification des espèces minérales d'après leurs compositions chimiques et leurs formes cristallines.

Empruntant la voie que Delafosse avait défrichée, Auguste Bravais développa la théorie des assemblages réticulaires et établit les lois mathématiques permettant de décrire l'ensemble des structures possédant des symétries d'orientations compatibles avec la triple périodicité des cristaux dans les trois dimensions de l'espace. II distingua ainsi 32 classes de symétrie réparties en 14 types de réseaux, que l'on peut regrouper en 7 systèmes définissant la forme de la maille élémentaire. Toujours dans la continuité des travaux de Delafosse, Pasteur fit sa mémorable découverte de la dissymétrie moléculaire. Cette dernière survint de la manière sui-

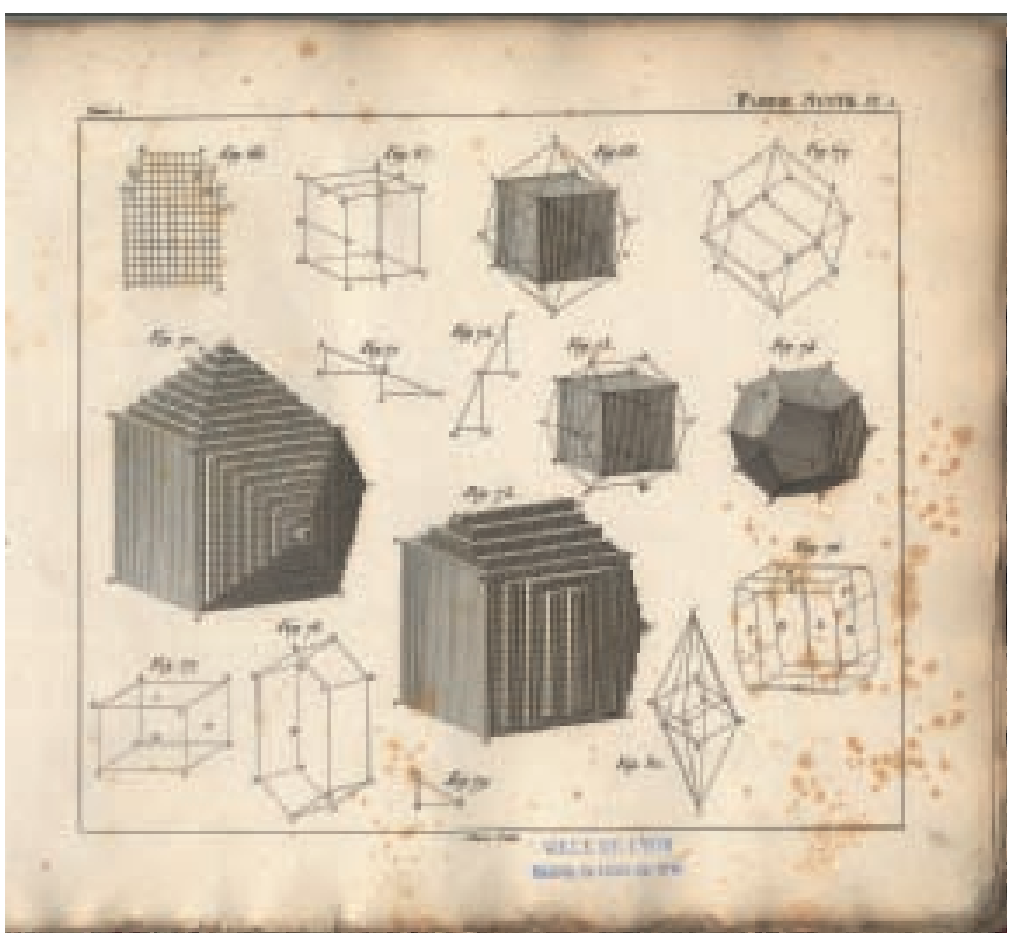

Figure 2. Représentation de la structure de quelques cristaux dans le Traité de cristallographie de René-Just Haüy (Bachelier et Huzard, Paris, 1822, planche 5 de l'atlas). L'empilement régulier des "molécules intégrantes " est bien visible. Cliché reproduit par les soins du Département du Fonds Ancien de la Bibliothèque Municipale de la Part-Dieu de Lyon. vante : examinant au microscope les cristaux d'un sel de l'« acide racémique », Pasteur montra que le corps étudié était en réalité un mélange de deux formes cristallines hémièdres, images l'une de l'autre dans un miroir. Les deux espèces, une fois isolées et mises en solution dans l'eau, manifestaient des pouvoirs rotatoires de même valeur absolue, mais de signes opposés. Quant au mélange initial, équimolaire, il était inactif sur la lumière polarisée. Pasteur déduisit de cela un principe fondamental : la dissymétrie cristalline résultait d'une dissymétrie moléculaire, elle-même à l'origine de l'isomérie optique.

La radiocristallographie confirma plus tard l'exactitude de la description du milieu cristallin réalisée par Haüy et Delafosse. Elle permit aussi de trancher définitivement la controverse sur l'isomorphisme.

\section{Des Cloizeaux et l'optique}

Le septième titulaire de la chaire de « Minéralogie » au Muséum, Alfred Des Cloizeaux (1817-1897), développa l'utilisation des méthodes optiques dans sa discipline. Élève d'Alexandre Brongniart au Muséum, de Dufrénoy et d'Henri de Sénarmont à l'École des Mines, il avait aussi fréquenté le laboratoire de Biot au Collège de France. Des Cloizeaux effectua, dans la grande tradition des « naturalistes voyageurs » du Muséum, des missions scientifiques dans plusieurs pays d'Europe, notamment en Islande où il étudia les gisements de spath biréfringent.

Si de Sénarmont saisit le premier tout l'intérêt de la lumière polarisée pour l'étude des cristaux, Des Cloizeaux fut le véritable propagateur de l'utilisation des méthodes optiques en minéralogie. Son prédécesseur dans la chaire de «Minéralogie » du Muséum, Gabriel Delafosse, avait d'ailleurs amorcé cette orientation en associant le phénomène de polarisation rotatoire à des caractéristiques de la structure interne des cristaux, comme l'hémiédrie. Des Cloizeaux décrivit les propriétés optiques de près de cinq cents minéraux transparents ou translucides, en utilisant des échantillons préparés par lui-même. II travailla durant vingt ans sur les feldspaths, dont il devint un spécialiste reconnu et mit en évidence le phénomène de polarisation rotatoire dans le cinabre. Avec l'optique, Des Cloizeaux fournit des critères efficaces pour définir l'« espèce minéralogique ». II fut également en mesure de résoudre des questions devant lesquelles la chimie ou la cristallographie s'étaient révélées impuissantes, comme l'analyse des cristaux de forme imparfaite : grâce à l'étude des propriétés optiques de ces derniers, le savant put déterminer leur type, donc les faire entrer dans des catégories précises. L'œuvre de Des Cloizeaux constitua par ailleurs le fondement de la lithologie moderne, en permettant de développer des classifications pétrographiques. Fouqué, Michel-Lévy et Lacroix devaient assurer la poursuite de ce travail.

Avant Des Cloizeaux, il n'existait pas de technique d'observation adaptée aux substances fournissant des lames très petites, d'une faible réfringence ou fragiles. Le savant voulut pallier cette carence vers 1857, en perfectionnant les microscopes polarisants d'Amici et de Nörrenberg (voir figure 3).

Enfin, Des Cloizeaux étudia de façon systématique les variations des propriétés optiques des minéraux sous l'action de la chaleur. II découvrit ainsi les changements d'écartement des axes optiques sous l'influence des variations de température. Tantôt l'action de la chaleur affecte seulement les caractères généraux du réseau cristallin, tantôt elle atteint le motif moléculaire inclus dans la maille : dans ce dernier cas s'opèrent les mutations profondes du dimorphisme, qui constitue l'un des phénomènes les plus importants de la physique moléculaire. Toujours dans le cadre de ses 
investigations sur l'influence de la chaleur, Des Cloizeaux désigna sous le terme de "pyrognomiques » les minéraux contenant des terres rares et devenant incandescents à une certaine température ${ }^{(1)}$. II étudia les propriétés optiques et pyrogénétiques de ces substances - comme l'orthite ou la gadolinite - en collaboration avec Damour. Au $20^{\mathrm{e}}$ siècle, il devait être démontré que le phénomène concerné se trouvait lié à la présence d'éléments radioactifs.

Les recherches optiques de Des Cloizeaux portèrent, nous l'avons dit, exclusivement sur les minéraux transparents, en raison de la nature même des méthodes employées, qui excluaient l'examen - même en sections minces - des composés opaques. Or, l'étude précise de ces derniers, constituants essentiels des minerais métalliques, offre un grand intérêt scientifique et industriel. Elle réclame la mise en œuvre d'une technique microscopique basée sur l'optique des cristaux opaques en lumière polarisée réfléchie. Avant-dernier titulaire de la chaire de « Minéralogie » du Muséum, Jean Orcel étendit en 1927 ce moyen d'investigation, grâce à la mise au point d'un microscope polarisant par réflexion. Un tel appareillage, obtenu par modification du microscope métallographique de Le Chatelier, permet de réaliser des observations qualitatives - couleur, dureté, clivage, teintes de polarisation sur des minéraux opaques.

\section{Électricité et magnétisme}

Afin de classer les espèces minérales, Haüy utilisa des critères reposant sur leurs propriétés électriques et magnétiques. Il se trouva ainsi conduit à faire des découvertes intéressantes. En 1817, il observa de manière qualitative l'émission d'électricité par un cristal de tourmaline ou de topaze soumis soit à une contrainte mécanique (piézoélectricité), soit à un échauffement (pyroélectricité). Haüy publia sur le sujet un mémoire intitulé « Sur l'usage des caractères physiques des minéraux, pour la distinction des pierres précieuses qui ont été taillées ». Antoine-César Becquerel prolongea ces travaux en étudiant la pyroélectricité des cristaux de tourmaline. II généralisa aussi à tous les corps les observations d'Haüy sur l'électrisation mécanique, mais sans recueillir l'assentiment de son collègue. II devait être démontré par la suite que le phénomène observé par les deux savants du Muséum n'était pas une manifestation piézoélectrique véritable, mais seulement une électrisation de surface. L'étude complète, théorique et expérimentale, de la vraie piézoélectricité devait être réalisée en 1880 par Pierre et Jacques Curie, auxquels on attribue la découverte de l'effet piézoélectrique direct. Un an plus tard, l'effet piézoélectrique inverse (ou réciproque), c'est-à-dire la déformation d'un cristal par l'action d'un champ électrique, fut mis en évidence grâce à des considérations thermodynamiques par Gabriel Lippmann et confirmé expérimentalement par les frères Curie. La piézoélectricité, qui se trouve directement liée à la symétrie cristalline, consiste en une polarisation de la maille élémentaire.

Haüy décrivit par ailleurs les propriétés magnétiques de nombreux minéraux. Approchant une aiguille aimantée d'un échantillon, il notait le sens et l'intensité de la déviation imprimée au petit aimant. Le critère magnétique, comme ses observations électriques (électricité produite par le frottement, pyroélectricité), optiques (couleur, densité, dureté, réfraction) ou mécaniques (densité, dureté), furent pris en compte par le savant pour construire des tableaux de classifications répertoriant un grand nombre de minéraux. Ces résultats classificatoires le firent désigner par Georges Cuvier comme le « législateur de la minéralogie ».

1. Tous les solides rougeoient quand on les chauffe ; mais ceux dits « pyrognomiques ", comme la gadolinite, rougeoient à beaucoup plus basse température. Ce terme a été introduit par Scheere en 1840, mais le phénomène avait été observé antérieurement par Wollaston et Berzelius

(http://en.wikipedia.org/wiki/Pyrognomic).
Il faut ajouter que le professorat d'Haüy constitua une véritable chance pour les collections cristallographiques du Muséum. En effet, la réputation du savant fit affluer dans l'établissement de nombreux échantillons, envoyés par des collègues ou des souverains étrangers. Haüy négocia par ailleurs l'acquisition du fonds Weiss et fabriqua des modèles cristallographiques en bois, que l'on peut toujours admirer au Muséum. Sa collection personnelle ne fut rachetée qu'en 1848 par l'établissement.

\section{Conclusion}

La physique développée dans la chaire de «Minéralogie » du Muséum participa largement à la définition du concept d'“ espèce minéralogique ", lequel se révéla fondamental pour le développement d'une discipline située au croisement des sciences de la matière et des sciences de la Terre. Jean Orcel a souligné ce point dans nombre de ses articles. Si l'on adopte une vision plus globale des événements, il est possible de considérer que la physique fut pratiquée de deux manières différentes par les minéralogistes dont nous avons analysé l'oeuvre. D'une part, selon une approche théorique, permettant de dégager des lois générales de la matière. Ce courant se trouve représenté par les travaux d'Haüy et de Delafosse, qui jouèrent un rôle de premier plan dans l'émergence de la cristallographie moderne. D'autre part, dans une perspective appliquée, en exploitant divers phénomènes phy-

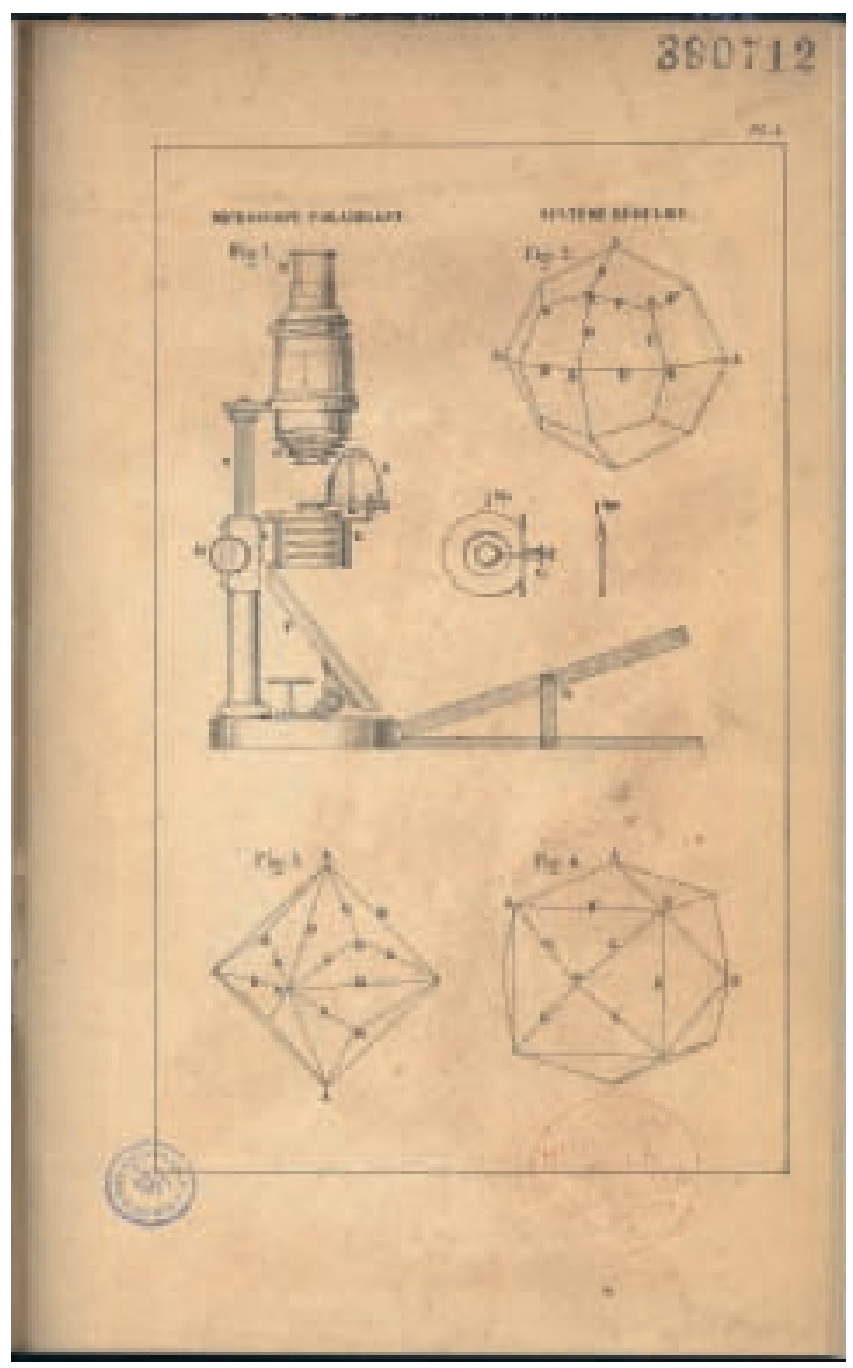

Figure 3. Le microscope polarisant de Des Cloizeaux (planche 1 du Manue de Minéralogie par A. Des Cloizeaux, Atlas, Dunod, Paris, 1862). Cliché reproduit par les soins du Département du Fonds Ancien de la Bibliothèque Municipale de la Part-Dieu de Lyon. 
siques pour décrire les minéraux, les distinguer les uns des autres et les classer. Les études optiques de Des Cloizeaux et d'Orcel, qui se traduisirent par la conception d'appareillages originaux - mais au domaine d'utilisation très ciblé -, appartiennent à ce second courant. Aujourd'hui, les conséquences plus ou moins immédiates de toutes ces investigations sont tangibles : la physique du solide, l'exploitation des gisements miniers, la géophysique, l'astrophysique et l'étude des structures anatomiques minérales des êtres vivants (coquilles, squelettes), en bénéficient très largement.

\section{Remerciements}

Nous tenons à remercier l'École nationale supérieure des Mines de Paris et la Bibliothèque municipale de la Part-Dieu de Lyon pour les clichés reproduits dans cet article.

\section{Bibliographie}

Balibar, F., La science du cristal, Hachette, Paris, 1991, 103 p.

Barbo, L., Les Becquerel, Belin, Paris, 2003, 142 p.
Jaussaud, P. et Brygoo, E., Du Jardin au Muséum en 516 biographies, Collection Archives, Muséum National d'Histoire Naturelle, Paris, 2004, 632 p.

Le Grand, Y., « La Tradition des Becquerel. Leçon inaugurale du cours de physique appliquée aux sciences naturelles, prononcée le 8 novembre 1949 ", Bull. Mus. natl. Hist. nat., $2^{\mathrm{e}}$ série, vol. 21, 1949 , pp. 648-662.

Orcel, J., " Histoire de la chaire de Minéralogie du Muséum national d'histoire naturelle ", Bull. Mus. natl. Hist. nat., $2^{\mathrm{e}}$ série, vol. 10, 1938 , pp. 328-354.

Orcel, J., "Les sciences minéralogiques ", in Taton R., Histoire générale des sciences, t. III, La science contemporaine, vol. I, Le XIX siècle, Presses Universitaires de France, Paris, 1961, pp. 343-369.

Pénicaud, A., Les cristaux, fenêtres sur l'invisible, Ellipses, Paris, 1999, 110 p.

Sadron, C., "La Biophysique moléculaire : leçon inaugurale du cours de biophysique, prononcée le 18 décembre 1963 ", Bull. Mus. natl. Hist. nat., $2^{\mathrm{e}}$ série, vol. 36, 1964, pp. 541-569.

Schnitter, C., "Le Muséum National d'Histoire Naturelle de Paris ; un terrain d'affrontement entre naturalistes et expérimentalistes ? ", Bull. Hist. Epistém. Vie, vol. 2, 1995, pp. 41-49.

\section{Les leçons de physique, chimie et histoire naturelle de l'École normale de l'an III}

\section{Etienne Guyon}

ESPCI, Paris

L'abbé René-Just Haüy, fondateur de la cristallographie géométrique, a également joué un rôle important dans l'enseignement de la physique au début du $19^{e}$ siècle, en particulier par les cours donnés aux 1400 normaliens de l'an III en 1794. Nous avons trouvé intéressant de compléter l'article ci-dessus de C. Kaspar et P. Jaussaud sur la "Physique et minéralogie au Muséum " par ce texte de E. Guyon, sur les cours de l'an III dont les "Leçons de physique, chimie et sciences naturelles "viennent d'être rééditées par les Presses de l'École normale supérieure.

Au tout début de la longue vie du Muséum, décrite dans l'article de C. Kaspar et P. Jaussaud, une courte aventure, pas si anecdotique que cela, mérite d'être contée : la création de l'École normale de l'an III, dans l'amphithéâtre Verniquet (le grand amphithéâtre du Muséum qui venait d'être construit), où étudièrent, de janvier à avril 1795, plus d'un millier " de citoyens déjà instruits " sous " les maîtres les plus habiles ". L'ambition de la Convention dans la création de cette École était de former un corps enseignant que l'on retrouvera les années suivantes dans les Écoles centrales (les écoles normales) afin d'éduquer tous les jeunes. Les cours furent donnés dans des conditions exceptionnelles. Les enseignements portaient sur l'ensemble des disciplines de l'esprit. Les cours étaient sténotypés et distribués aux élèves quelques semaines plus tard; les débats étaient sténotypés... La mission du Bicentenaire de la Révolution, que présidait $\mathrm{J}$. N. Jeanneney, recommanda fort justement l'édition de cet ensemble considérable, qui est un témoignage des savoirs à la fin du Siècle des Lumières. Les leçons de mathématiques données par Laplace, Lagrange et Monge, qui influencèrent l'enseignement des mathématiques au $19^{\mathrm{e}}$ siècle et celles de Sciences Morales et Politiques furent ainsi édités par Dunod au début des années 1990 .

Les leçons de physique, de chimie et d'histoire naturelle viennent d'être éditées aux Presses de l'École normale supérieure, rue d'Ulm (http://www.presses.ens.fr ). Elles rassem- sous la direction

d'ÉTIENNE GUYON

L'ÉCOLE NORMALE DE L'AN III LEÇONS DE PHYSIQUE DE CHIMIE D'HISTOIRE NATURELLE

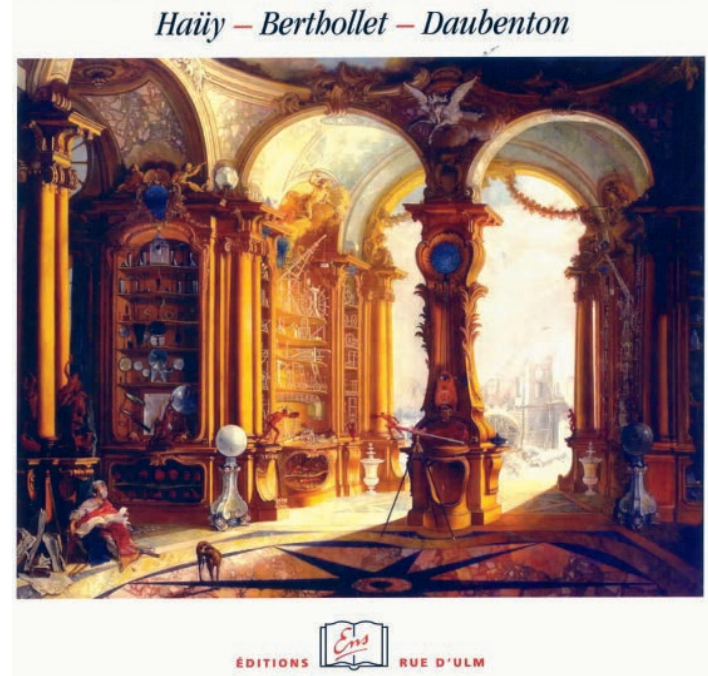

Page de couverture de la nouvelle édition des Leçons de phy sique, chimie et histoire naturelle éditées par les Presses de l'ENS. blent les cours du jeune Berthollet en chimie, de Daubenton, alors directeur âgé du Muséum, et de l'abbé Haüy en physique, qui avait suivi les cours de Daubenton au Muséum. Arrêtons-nous un instant sur le cours de ce physicien qui, le rappelle le texte précédent, fut ensuite professeur au Muséum. L'édition critique de ce très important cours est le résultat du travail de Nicole Hulin, Bernard Maitte pour la cristallographie, Christine Blondel pour l'électricité, et Michel Blay. II s'agit d'un document très complet sur les savoirs en sciences physiques de l'époque, ouvert sur les savoirs " modernes » (Newton, Coulomb) et qui fait naturellement une grande part à la cristallographie. Le cours de Haüy, sous une forme pas très modifiée, sera utilisé dans les programmes d'enseignement dans toute la première partie du $19^{\mathrm{e}}$ siècle.

L'édition de cette série d'ouvrages sera complétée par une "prosopographie "(1) des normaliens, due à l'historien Bernard Julia, qui a identifié le parcours de la majorité des élèves de cette éphémère mais essentielle École. L'édition des cours littéraires est aussi programmée.
1. Prosopographie : terme couramment utilisé par les historiens pour une biographie collective. 